\title{
In-Situ Determination of Critical Welding Stresses During Assembly of Thick-Walled Components made of High-Strength Steel
}

\author{
Dirk Schroepfer ${ }^{1, a,{ }^{*}}$, Arne Kromm ${ }^{1, b}$, Andreas Hannemann ${ }^{1, \mathrm{c}}$ and \\ Thomas Kannengiesser ${ }^{1, d}$ \\ ${ }^{1}$ Bundesanstalt für Materialforschung und -prüfung (BAM), Unter den Eichen 87, 12205 Berlin, \\ Germany. \\ adirk.schroepfer@bam.de, barne.kromm@bam.de, 'candreas.hannemann.de, \\ dthomas.kannengiesser.de
}

Keywords: Residual Stress, Welding, Large-Scale Test, X-Ray Diffraction, HSLA Steel

\begin{abstract}
The performance and safety of welded high-strength low-alloyed steel (HSLA) components are substantially affected by the stresses occurring during and after welding fabrication, especially if welding shrinkage and distortion are severely restrained. The surrounding structure of the whole component affects loads in the far-field superimposing with welding stresses in the near-field of the weld. In this study a unique testing facility was used to restrain shrinkage and bending while analyse multiaxial far-field loads (max. $2 \mathrm{MN}$ ) during assembly of thick-walled component. A novel approach for the assessment of the in-situmeasured far-field data in combination with the actual weld geometry was elaborated. For the first time, analyses of the global bending moments of restrained welds based on the neutral axis of the actual weld load bearing section were achieved. Hence, far-field measurements offered the possibility to determine critical near-field stresses of the weld crosssections for the entire joining process. This work presents the approach for far-to-near field in-situ determination of stresses in detail for the 2-MN-testing system based on an extensive experimental work on HSLA steel welds, which demonstrates sources and consequences of these high local welding stresses. Thus, it was clarified, why the first weld beads are crucial regarding welding stresses and cold cracking, which is well known, but has never been measured so far. Accompanying analyses using X-ray diffraction (XRD) after welding show effects on local residual stress distributions. These analyses indicated viable prospects for stress reduction during assembly of thick-walled HSLA steel components.
\end{abstract}

\section{Introduction}

Residual stresses in welds are the result of the inhomogeneous volume changes due to welding process, cooling and material behaviour [1,2]. Especially in HSLA steels high tensile residual stresses are crucial due to the high yield ratio and low plastic strain reserves as well as the challenging and limited parameter range (i.e. cooling time) for the welding processes. An improved knowledge of evolution, distribution and magnitude of the arising residual stresses may obviate failure during manufacturing or even service. Welding process optimization considering stressing even enables enhancements of safety and performance (strength, toughness, fatigue, cracking resistance etc.) of HSLA steel welds and a sustainable, economic utilization of the material $[2,3]$. Studies and standardized weld tests involving stresses predominantly focus on local residual stresses in free shrinking laboratory size specimens [4]. Realistic heat conduction and the rigidity of the oftentimes thick-walled component welds are usually not taken into consideration. Generally, during component welding, shrinkage is hindered additionally to the local restraint in the weld and its vicinity as a result of global shoring effects and stiffness of the 
surrounding structure [5]. The arising far-field reaction forces and stresses by reasons of global restraint superimpose with local restraint stresses due to welding process and material behaviour, see Fig. 1a. Current studies reveal that the far-field loads may increase dependent on the stiffness of the structure, the welding process conditions and the applied filler and base materials, and critically prepossess also the near-field residual stress level in the weld seam $[3,6]$.
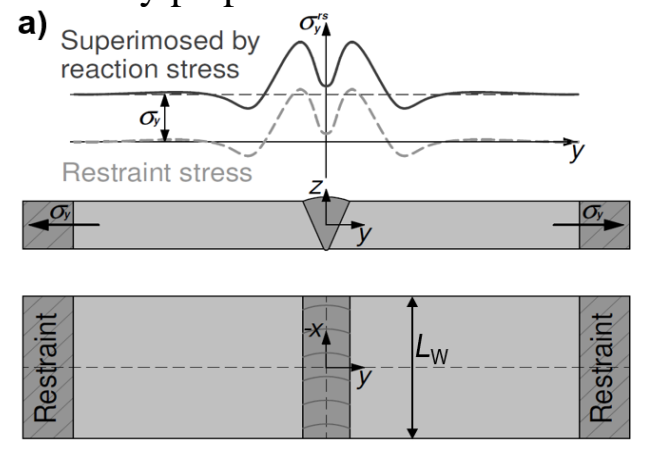

Fig. 1. Schematic of superposition of near and far-field loads (a) due to restraint of lateral shrinkage (b)/ angular distortion (c) with formula for restraint intensity factors $R_{F y}(E q .1) / R_{M x}$ (Eq. 2) [3].

For quantification for the stiffness of the weld seam towards the circumjacent construction, the restraint intensity factors $R_{\mathrm{Fy}}$ (lateral restraint, Fig. 1b) and $R_{\mathrm{Mx}}$ (bending restraint, Fig. 1c) based on the seam length $L_{\mathrm{W}}$ were established by [5]. Recently, numeric and experimental works dealt with the impact of restraint intensity on the residual stress state after welding and cooling of HSLA steel components [6-8]. However, numerical analysis of residual stress evolution in component-related welds are challenging and may lead to rash misinterpretation [9]. Besides, severe effects are also expected for the weld seam during the assembly, especially if highly restrained angular distortion occurs. Primarily, the first passes of HSLA steel welds are critical in the face of crack initiation due to high welding stresses. This phenomenon is well-known. Systematic measurements and detailed analyses to quantify these stresses and the influence of the boundary process parameters, material and realistic restraint conditions are absent, since insitu analyses of local stresses while welding are not feasible directly by conventional testing methods. At BAM, a unique testing facility was developed $[3,10]$, with which a defined restraint of both the lateral shrinkage and the angular distortion is achievable at the amount of real HSLA steel components. Simultaneously to the restraint, the multiaxial far-field loads can be analysed in-situ during welding experiments dependent on the applied welding heat control.

\section{Experimental}

Material and Welding Parameters. For the experiments, $H=20 \mathrm{~mm}$ thick plates of HSLA S690QL (EN 10025-6 [11]) were multilayer MAG-welded with matching solid wire of same type using typical parameters for HSLA steel application (i.e. mobile cranes), see Table 1. A pair of plates were clamped hydraulically into the testing facility and tack welded. Additional to the welding data, temperatures of the weld and weld vicinity were measured using thermocouples and a two-colour pyrometer. For comparison of XRD data and verification of required weld properties, free shrinking samples were welded as well. Cooling times $\left(t_{8 / 5}\right)$ were appropriate to steel producer recommendations for test no. 1 to $4\left(t_{8 / 5}=11\right.$ to $\left.13 \mathrm{~s}\right)$. All tested free shrinking specimens with adequate weld heat control had suitable weld properties, except test no. 5 due to $t_{8 / 5}$-times being much higher than recommended $\left(t_{8 / 5}>20 \mathrm{~s}\right)$.

Restraint Intensities. Fig. 2 shows a schematic of the testing facility and the XRD measurements at the clamped specimens. With the formulas for restraint intensity factors (Eq. 1 and Eq. 2) the rigidity of the test weld could be quantified and compared to typical values for restraint conditions in case of HSLA steel application, Table 2 [5,8]. The restraint intensity $R_{\mathrm{Fy}}$ 
was discretised as shown in Eq. 3 for the test system $R_{\mathrm{Fy}, 2 \mathrm{MN}}$, from the experimental determined spring rigidity of the three-legged test frame $\left(C_{\mathrm{y}}\right)$ [10], and the plate/seam geometry $R_{\mathrm{Fy}, 12}$ calculated from the specimen stiffness [8]. The bending restraint $R_{\mathrm{Mx}}$ is the result of Eq. 4 with lever arms of $a=230 \mathrm{~mm}$ from the neutral axis of the specimen to the upper and lower piston rods of the testing facility. For the free shrinking specimens, the restraint intensity factors are both zero, since there is no stiffness of the weld against any surrounding structure. Note that due to bisect of the weld length $L_{\mathrm{W}}$ the lateral restraint intensity of the test setup would increase by $30 \%$, whereas the bending restraint intensity would almost double.

Table 1. Chemical composition (FES, Fe balanced), mechanical properties of the $20 \mathrm{~mm}$ thick test plates and welding parameters of the weld tests.

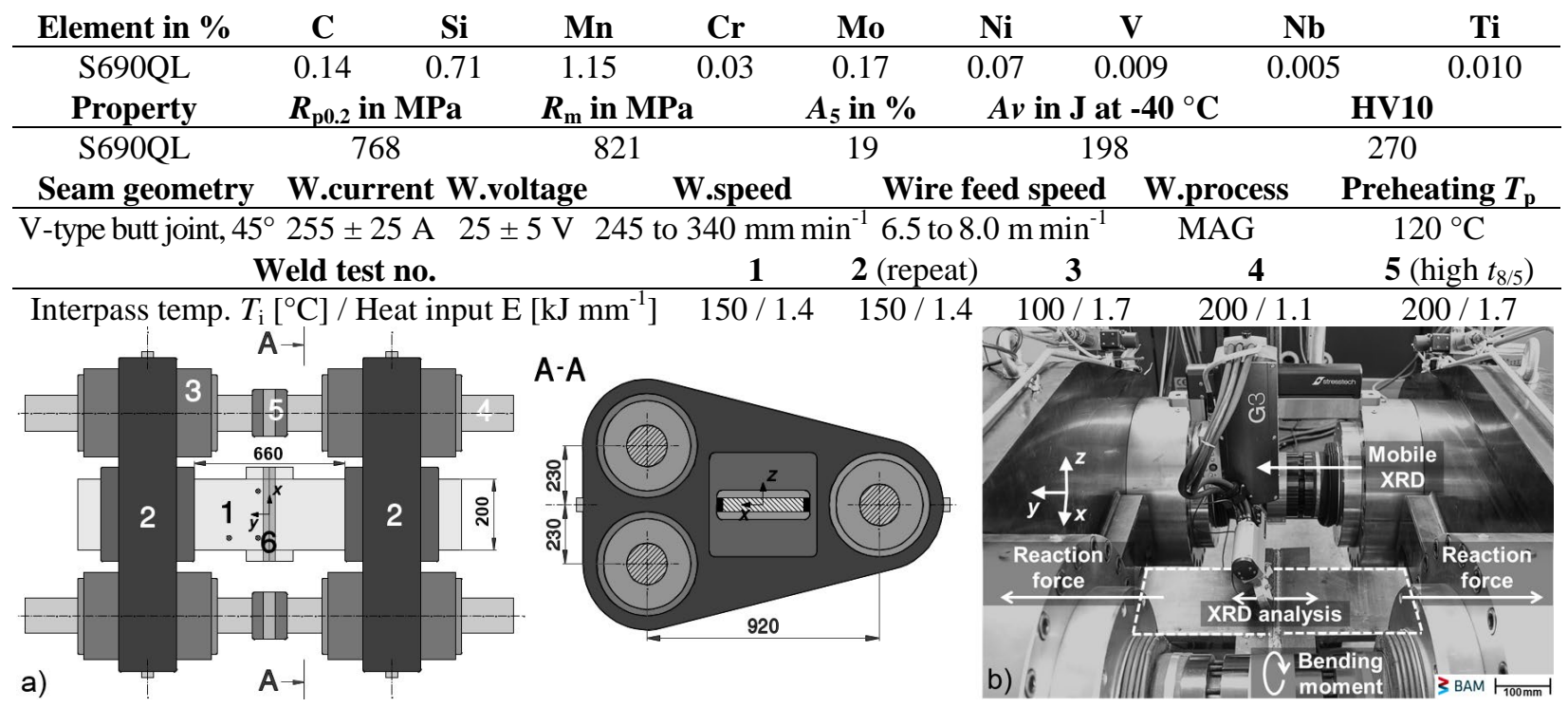

Fig. 2. a) Schematic of 2-MN-testing facility (1-specimen, 2-test desk, 3-hydraulic system, 4piston rod, 5-force measurement, 6-weld seam); b) residual stress analysis in the testing facility [8].

Table 2. Restraint intensity factors for different test setups $\left(C_{y, t e n s, c y l}=407.3 \mathrm{kN} \mathrm{mm}^{-1} ; C_{y, \text { comp,cyl }}\right.$ $\left.=459.0 \mathrm{kNmm}^{-1}\right)$.

Test setup Weld length Plate length System restraint Specimen restraint Lateral restraint Bending restraint

\begin{tabular}{ccccccc}
- & $L_{\mathrm{W}}[\mathrm{mm}]$ & $L_{\mathrm{E}}[\mathrm{mm}]$ & $R_{\mathrm{Fv}, 2 \mathrm{MN}}\left[\mathrm{kN}(\mathrm{mmmm})^{-1}\right]$ & $R_{\mathrm{Fy}, 12}\left[\mathrm{kN}(\mathrm{mmmm})^{-1}\right]$ & $R_{\mathrm{Fy}}\left[\mathrm{kN}(\mathrm{mmmm})^{-1}\right]$ & $R_{\mathrm{Fy}}\left[\mathrm{kNm} /\left({ }^{\circ} \mathrm{mm}\right)^{-1}\right]$ \\
\hline Free shrinking & 200 & 150 & 0 & 0 & 0 & 0 \\
2-MN-test fac. & 200 & 330 & 6.1 & 5.8 & 3.0 & 8
\end{tabular}

$$
\begin{aligned}
& R_{\mathrm{Fy}}=\left(R_{\mathrm{Fy}, 2 \mathrm{MN}} \cdot R_{\mathrm{Fy}, 12}\right) /\left(R_{\mathrm{Fy}, 2 \mathrm{MN}}+R_{\mathrm{Fy}, 12}\right) . \\
& R_{\mathrm{Mx}}=\left\{\mathrm{a}^{2} \cdot \tan \Delta \beta \cdot\left(C_{\mathrm{y}, \mathrm{comp}, \mathrm{cyl}}+C_{\mathrm{y}, \mathrm{tens}, \mathrm{cyl}}\right)\right\} /\left\{2 \Delta \beta L_{\mathrm{W}}\right\} .
\end{aligned}
$$

\section{Results}

Global Forces. Fig. 3a depicts reaction forces $F_{\mathrm{y}}(t)$ while preheating, welding and cooling of the two weld tests no. 1 and 2, welded under the same boundary conditions, welding parameters and recommended heat control (medium $T_{\mathrm{i}}$ and $E$ ). As a result, both graphs are qualitatively and quantitatively almost equal with a little scatter range in-between. After some compression while preheating, during root welding, the already solidified inserted weld metal produces transversal shrinking forces, which increase further to a first maximum of $F_{\mathrm{y}}=62 \mathrm{kN}$ and $M_{\mathrm{x}}=0.5 \mathrm{kNm}$ during cooling to $T_{\mathrm{i}}=150{ }^{\circ} \mathrm{C}$. Forces decrease while welding of the second layer due to the local heat input combined with stress relief. Subsequent cooling to $T_{\mathrm{i}}$ leads to a growth of reaction force, the next weld run to a transient reduction. This evolution repeats for each weld sequence. Reaction force amplitudes increase with each weld layer due to welding heat input. Subsequent 
cooling to room temperature (RT) after the cap bead leads to a maximum force of $F_{\text {y,end }}=535 \mathrm{kN}$.

Bending Moments. Restraint angular distortion of welds cause a disequilibrium of reaction forces over the plate thickness direction, resulting in a reaction moment $M_{\mathrm{x}}$ around the neutral axis of the weld in $x$ - direction [8].
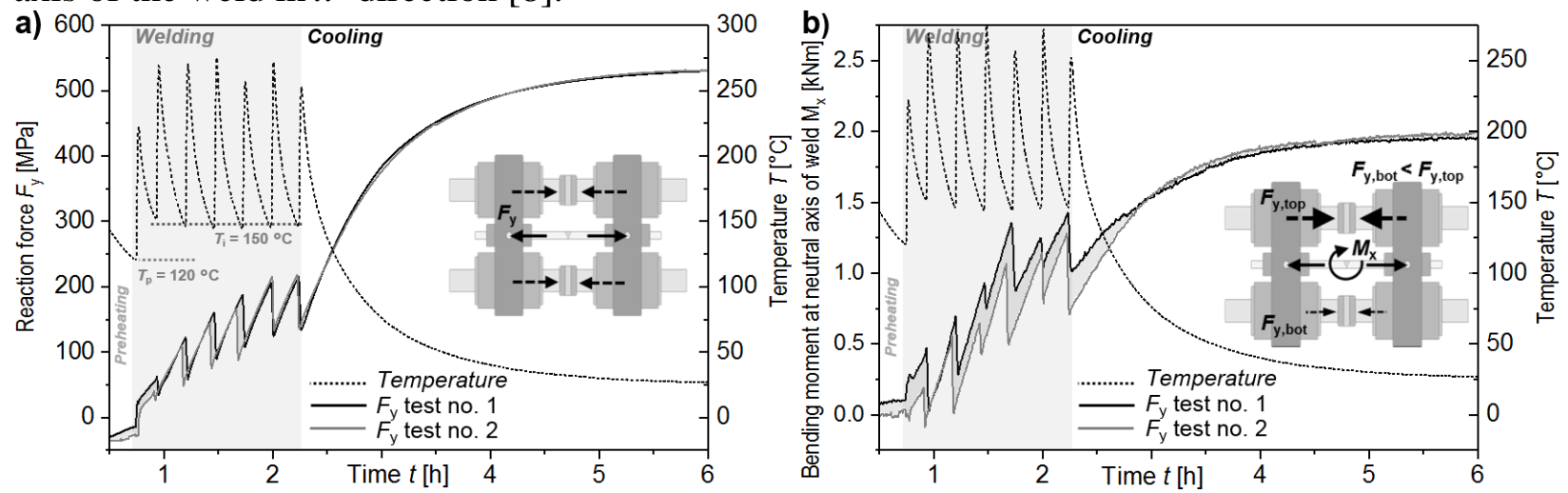

Fig. 3. Reaction forces $F_{y}(t)$, temperature $T(t)(a)$ and Bending moments $M_{x}(t)(b)$ of two weld tests with same parameters, 2-MN-testing facility $\left(T_{p}=120^{\circ} \mathrm{C}, T_{i}=150{ }^{\circ} \mathrm{C}, E=1.4 \mathrm{~kJ} \mathrm{~mm}^{-1}\right)$.
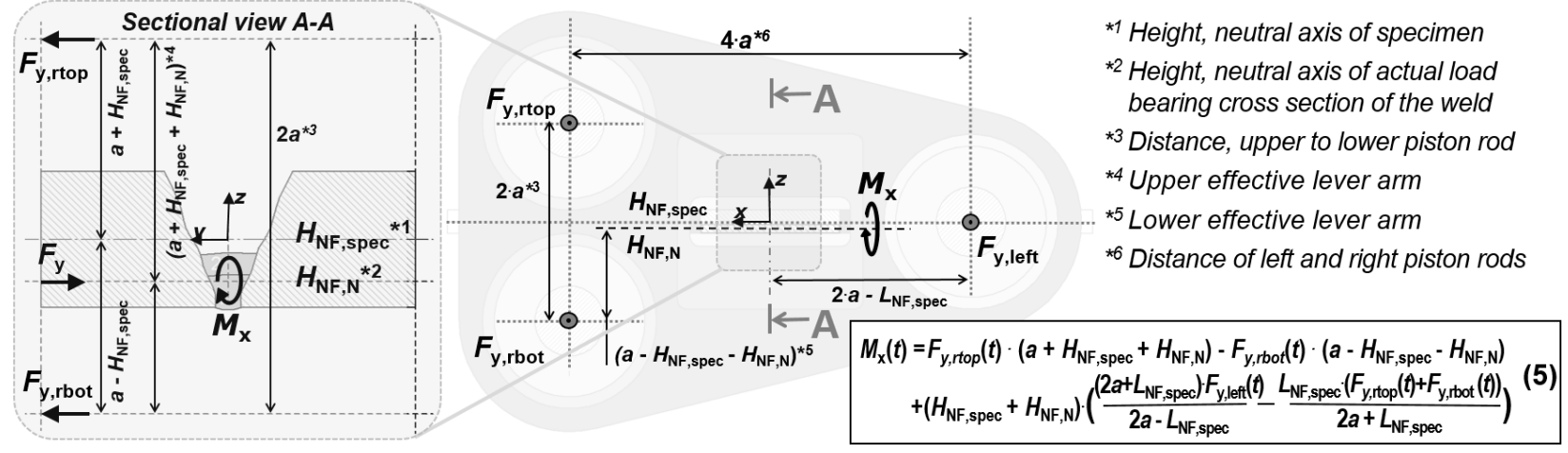

Fig. 4. Determination of the bending moments $M_{x}(t)$ based on the neutral axis of the actual load bearing cross section of the weld in the 2-MN-testing facility [8].

For detection of this bending moment $M_{\mathrm{x}}$ while welding, the height of the neutral axis of the specimen $H_{\mathrm{NF} \text {,spec }}$ and of the actual load bearing cross section $H_{\mathrm{NF}, \mathrm{N}}$ of the weld have to be known, see Fig. 4. With the determined upper and lower lever arms of the proof frame and a compensation regarding the neutral axis of the specimen towards the left piston rod and force measurements of the hydraulic system, finally, the actual bending moment $M_{\mathrm{x}}(t)$ can be calculated, using Eq. 5, shown exemplarily for the two medium parameter weld tests (no. 1 and 2 ) in Fig. 3b. It is obvious that the $M_{\mathrm{x}}(t)$-graphs are qualitatively equal to the $F_{\mathrm{y}}(t)$-graphs, since the force above the neutral axis of the weld is ever higher than below. The scatter band between the two $M_{\mathrm{x}}(t)$-graphs of weld test no. 1 and 2 is slightly broader compared to the $F_{\mathrm{y}}(t)$-graphs, which reflects the higher susceptibility to errors, i.e. due to metallographic analyses of the weld layers at metallographic cross section. The maximum bending moment of both weld tests occur after cooling to RT at approx. $M_{\mathrm{x}, \text { end }}=2 \mathrm{kNm}$.

Far-field Loads. Reaction forces due to welding result in global stresses at the weld seam, which can be calculated and in-situ analysed, see Fig. 5, cf. Fig. 3. Constant forces over the weld seam thickness lead to normal reaction stress. The magnitude depends on the load bearing section of the weld, see Fig. 5a. Transient stress increases significantly while cooling to interpass temperature already after root welding due to the comparable small load bearing cross section area. It could be observed that the heat input and phase transformation of the weld metal at the next weld runs leads to a transient stress reduction and a new stress increase during cooling to $T_{\mathrm{i}}$. 
Compared to the $F_{\mathrm{y}}(t)$-graphs, there is, hence, a smaller difference between the stress levels while welding and after cooling to RT, especially if low interpass temperatures were applied $[3,8]$.
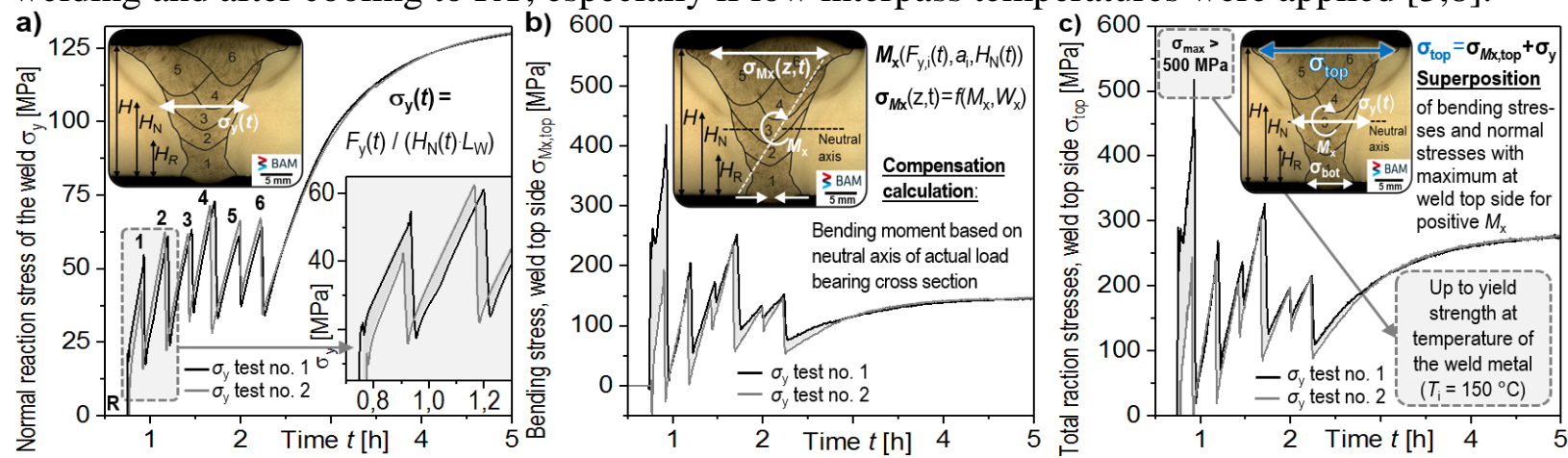

Fig. 5. Normal stress $\sigma_{y}(t)(a)$, bending stresses $\sigma_{M x}(t)(b)$, total stress at weld top side $\sigma_{\text {top }}(t)(c)$ of two weld tests with same parameters, 2-MN-testing facility $\left(T_{p}=120^{\circ} \mathrm{C}, T_{i}=150^{\circ} \mathrm{C}, \mathrm{E}=1.4 \mathrm{~kJ}\right.$ $\left.m m^{-1}\right)$.

$\sigma_{\mathrm{Mx}}(t)=M_{\mathrm{x}}(t) / W_{\mathrm{x}} \quad$ with $W_{\mathrm{x}}=L_{\mathrm{W}} \cdot H^{2} / 6$.

Near-field Loads. A positive bending moment arising during welding and cooling of the restrained weld, causes bending stresses, which concentrate on the top side of the weld. With in-situ bending moment analyses based on the neutral axis of the load bearing cross section the calculation of the actual local bending stresses on the weld top side $\sigma_{\mathrm{Mx}}(t)$ was possible for the first time for the whole welding process of HSLA steels, see Eq. 6 and Fig. 5b. A superposition with the normal reaction stresses leads to total reaction stresses at the top side of the weld, Fig 5c. It was observed that especially cooling of the root weld already at medium interpass temperature leads to high stresses exceeding $500 \mathrm{MPa}$, the yield strength at temperature for the microstructure of the weld metal, and the total stresses of the complete weld after cooling to RT. Also, the superimposing bending stresses of approx. $\sigma_{\mathrm{Mx}}=150 \mathrm{MPa}$ cause almost $300 \mathrm{MPa}$ total end reaction stresses $\sigma_{\text {top,end. }}$
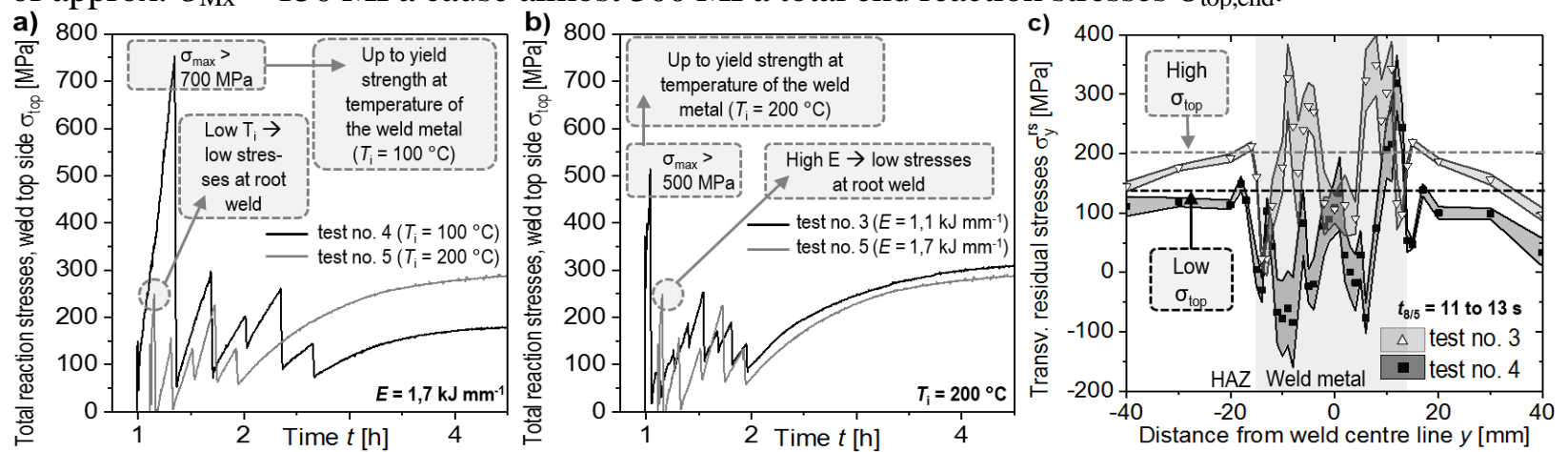

Fig. 6. Total stress at weld top side $\sigma_{\text {top }}(t)$ for different interpass temperature (a) and heat input

(b); comparison of transverse residual stress distributions of two weld tests with different total stress levels.

Heat control affects the arising welding stresses significantly, see Fig. 6. A low interpass temperature leads to lower end reaction stresses, as observed also in $[3,8,10]$, b ut high total stresses at the top of the root weld due to longer cooling phases compared to higher $T_{\mathrm{i}}$, with stresses exceeding the yield strength of the weld metal. A lower heat input at high $T_{\mathrm{i}}$ results also in higher root stresses reaching the yield strength at temperature due to a smaller root section area. Somewhat higher total end reaction stresses occur. XRD analysis of the local residual stresses depicts Fig. 6c for the two welds with varied heat control and appropriate $t_{8 / 5}$-time (11 to $13 \mathrm{~s}$, test no. 3 and 4). The welding stress level is significantly increased if high $T_{\mathrm{i}}$ and low $E$ (test no. 3) were applied. Especially in the HAZ the increase is at the amount of the difference between the total end reaction stresses at the top of the weld (approx. 100MPa), cf. Fig. 6a and b. 


\section{Summary}

With a unique testing facility at BAM, component related weld test of a HSLA steel were performed using application related parameters and boundary conditions. Multiaxial far-field loads (max. $2 \mathrm{MN}$ ) during multilayer welding could be analysed. Special compensation calculation based on the neutral axis of the actual load bearing section allowed for the first time in-situ analyses of local welding stresses on the top of the weld. Coming from medium values for heat control, heat input and working temperature should be adapted together to reach recommended cooling time values of the weld. It could be shown that low heat and high working temperature lead to significantly higher welding stresses. Low working temperature with increased heat input enables low welding stresses and low local residual stresses especially in the HAZ, but leads to high welding stresses during root weld's cooling to low interpass temperature, which is crucial for the whole welding process and, hence, weld integrity. Special procedures for root welding to prevent high root stresses could be: ductile filler materials, adapted working temperature strategy or higher deposition rate as well as adapted seam geometry to increase the cross section area and section modulus of the root [8]. Further investigations of such adapted concepts would help to enhance performance of HSLA steel welds and further utilization of the materials.

\section{References}

[1] T. Nitschke-Pagel, H. Wohlfahrt, The Generation of Residual Stresses due to Joining Processes, in: V. Hauk, H. Hougardy, E. Macherauch (Eds.), Residual Stress. - Meas. Calc. Eval., DGM Informationsgesellschaft mbH, 1991, pp. 121-133.

[2] P.J. Withers, H.K.D.H. Bhadeshia, Residual Stress. Part 2 - Nature and Origins, Mater. Sci. Technol. 17 (2001) 366-375. https://doi.org/10.1179/026708301101510087

[3] D. Schroepfer, A. Kromm, T. Kannengiesser, Engineering approach to assess residual stresses in welded components, Weld. World. 61 (2017) 91-106. https://doi.org/10.1007/s40194016-0394-9

[4] T. Kannengiesser, T. Boellinghaus, Cold cracking tests - An overview of present technologies and applications, Weld. World. 57 (2013) 3-37. https://doi.org/10.1007/s40194012-0001-7

[5] K. Satoh, Y. Ueda, H. Kihara, Recent Trends of Research into Restraint Stresses and Strains in Relation to Weld Cracking, Weld. World. 11 (1973) 133-156.

[6] D. Schroepfer, A. Kromm, T. Kannengiesser, Load analyses of welded high-strength steel structures using image correlation and diffraction techniques, 62 (2018) 459-469.

[7] M. Hirohata, Y. Itoh, Effect of Restraint on Residual Stress Generated by Butt-welding for Thin Steel Plates, in: 9th Ger. Bridg. Symp. Kyoto, Japan, 2012, pp. 1-6.

[8] D. Schröpfer, Adaptierte Wärmeführung zur Optimierung schweißbedingter Beanspruchungen und Eigenschaften höherfester Verbindungen, Dissertation, OVGU Magdeburg, Shaker Verlag Aachen, 2017.

[9] J. Klassen, T. Nitschke-Pagel, K. Dilger, Challenges in the Calculation of Residual Stresses in Thick-walled Components, in: Mater. Res. Proceedings, Residual Stress. 2016 ICRS-10, Materials Research Forum, 2016: pp. 299-304.

[10]T. Lausch, T. Kannengiesser, M. Schmitz-Niederau, Multi-Axial Load Analysis of ThickWalled Component Welds made of 13CrMoV9-10, J. Mater. Process. Technol. 213 (2013) 1234-1240. https://doi.org/10.1016/j.jmatprotec.2013.01.008

[11]EN 10025-6: Hot rolled products of structural steels - Part 6: Technical delivery conditions for flat products of high yield strength structural steels in the quenched and tempered conditions, 2018. 